\title{
Distribution Network Planning and Design Using Branch and Bound Methods
}

\author{
Jalal Abdallah \\ Tafila Applied University College, P.O. Box 851229, code 11185, Amman, Jordan
}

\begin{abstract}
This study presents implementation of the bound and branch methods as an optimization mathematical device for distribution network planning. A development technology concentrates on minimizing the total costs and provides extended opportunities for improvement of network operation, from the initial planning stage. The study illustrate the mathematical and the algorithm of the branch and bound method with an example to indicate the efficiency of the branch and bound in planning and design processes. It also shows that the optimal configuration strongly depends on the branching rule and on the bound calculation bases.
\end{abstract}

Key words: Branch and Bound Methods, Distribution Networks, Design, Planning

\section{INTRODUCTION}

The design of distribution networks starts with a map of the area, the positions of the roads, streets and buildings and position of distribution stations. They can be overhead or underground.

The electrical power system equipment's design is governed by the electrical and mechanical needs. The electrical design of distribution facilities is based on the loads that they are to carry safely and the permissible voltage variation. The configuration has a great effect on the optimization of the load flow in the system. The final design cannot be divorced from the mechanical, economic, and other considerations. Several different designs may serve the same electrical requirements adequately. Each, in turns, may be modified by mechanical considerations. The selected design ultimately must reflect the economic considerations, specifically, the design that results in the least annual expense in supplying the load or loads question. This necessarily involves the evolution of losses, as well as capital, maintenance, and operation expenses. Often, other considerations must also be taken into account including government regulations, national and local industry constrictions and safety codes, taxes, public relations and some other, intangible requirements [1]. Planing and development of distribution networks pursues a number of conflicting objectives: minimization of power losses, capital investments, operation and maintenance costs and energy not supplied due to interruption in the network. Multiple objectives, large number of variables, uncertainty of initial information and dynamic nature of the problem cause the complexity of the stated task.

The approximate hierarchy of objectives for distribution networks planning is presented. In Fig. 1 more or differently formulated objective can be added i.e voltage quality and environmental impact [2-4].

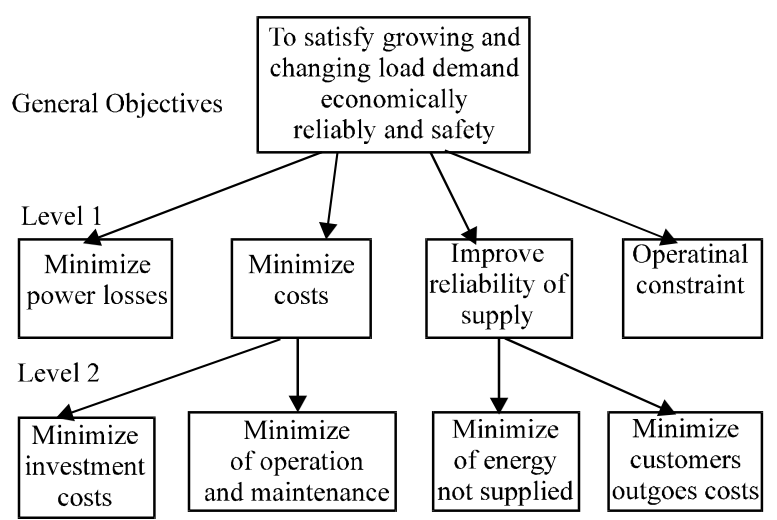

Fig. 1: The Hierarchy of Objectives for Distribution Networks Planning and Design

The available investment and the degree of reliability of supply govern the selection of the final type of the distribution configuration (whether radial, open loops or network). In low density urban areas and rural areas radial distributors are used. The medium and highdensity areas are generally supplied through open loop distributors. In the urban areas of developed countries are generally fed by network distribution schemes [5-7]. A great number of works and papers were presented in optimization the distribution system using different computational methods. Vaziri and Turkay $[8,9]$ have made survey and categorizing to large number of the previous works.

The Branch and bound methodologies are recommended to be used to find the optimal design configuration for the distribution networks, and improve the efficiency of planning process [6].

The Branch and Bound Methods: Branch and Bound methods are considered as a general search method. Suppose we wish to minimize a function $\mathrm{f}(\mathrm{x})$, where $\mathrm{x}$ 
is restricted to some feasible region (defined, e.g., by explicit mathematical constraints). To apply branch and bound methods, one must have a means of computing a lower bound on an instance of the optimization problem and a means of dividing the feasible region of a problem to create smaller subproblems. There must also be a way to compute an upper bound (feasible solution) for at least some instances; for practical purposes, it should be possible to compute upper bounds for some set of nontrivial feasible regions.

The method starts by considering the original problem with the complete feasible region, which is called the root problem. The lower-bounding and upper-bounding procedures are applied to the root problem. If the bounds match, then an optimal solution has been found and the procedure terminates. Otherwise, the feasible region is divided into two or more regions, each stricts subregions of the original, which together cover the whole feasible region; ideally, these subproblems divide the feasible region. These subproblems become children of the root search node. The algorithm is applied recursively to the subproblems, generating a tree of subproblems. If an optimal solution is found to a subproblem, it is a feasible solution to the full problem, but not necessarily globally optimal. Since it is feasible, it can be used to prune the rest of the tree: if the lower bound for a node exceeds the best known feasible solution, no globally optimal solution can exist in the subspace of the feasible region represented by the node. Therefore, the node can be removed from consideration. The search proceeds until all nodes have been solved or pruned, or until some specified threshold is met between the best solution found and the lower bounds on all unsolved subproblems.

The Mathematical Basics: The Branch and bound methods find the global minimum of function:

$$
\mathrm{f}: \mathrm{R}^{\mathrm{m}} \rightarrow \mathrm{R}
$$

over an $\mathrm{m}$ dimensional rectangle $\mathrm{Q}_{\text {init }}$, and by replacing $\mathrm{f}$ by-f, it can also be used to find the global maximum. For rectangle $\mathrm{Q} \subseteq \mathrm{Q}_{\text {init }}$ we define

$$
\varphi_{\min }(Q)=\min _{q \in Q} f(q)
$$

Then we define the $\phi_{\min }\left(\mathrm{Q}_{\text {init }}\right)$, which should be within an absolute accuracy of $\varepsilon>0$, using two functions $\phi_{\mathrm{lb}}(\mathrm{Q})$ and $\phi_{\mathrm{ub}}(\mathrm{Q})$ defined over $\left\{\mathrm{Q}: \mathrm{Q} \subseteq \mathrm{Q}_{\text {init }}\right\}$ which , presumably, are easier to compute than $\phi_{\min }(\mathrm{Q})$.These two functions must satisfy the following conditions :

$$
\text { - } \phi_{\mathrm{lb}}(\mathrm{Q}) \leq \phi_{\min }(\mathrm{Q}) \leq \phi_{\mathrm{ub}}(\mathrm{Q})
$$

Where, $\phi_{\mathrm{lb}}(\mathrm{Q})$ and $\phi_{\mathrm{ub}}(\mathrm{Q})$ are the lower and the upper bound, respectively.

As the maximum half- length of the sides of $\mathrm{Q}$, denoted by size (Q), goes to zero the difference between upper and lower bound converges to zero, i.e.,

$\forall \varepsilon>0 \exists \delta>0$

Such that $\forall \mathrm{Q} \subseteq \mathrm{Q}_{\text {init }}$, size

$(\mathrm{Q}) \leq \delta \Rightarrow \phi_{\mathrm{ub}}(\mathrm{Q})-\phi_{\mathrm{lb}}(\mathrm{Q}) \leq \varepsilon$.

Roughly speaking, then the bounds $\phi_{\mathrm{lb}}$ and $\phi_{\mathrm{ub}}$ become sharper as the rectangle shrinks to a point.

Branch and Bound Algorithm: According the mentioned mathematical model the Branch and Bound algorithm in general form can be described as [7]:

$$
\begin{aligned}
& \mathrm{K}=0 ; \\
& \mathfrak{I}_{0}=\left\{\mathrm{Q}_{\text {init }}\right\} ; \\
& \mathrm{L}_{0}=\phi_{\mathrm{lb}}\left(\mathrm{Q}_{\text {init }}\right) ; \\
& \mathrm{U}_{0}=\phi_{\mathrm{ub}}\left(\mathrm{Q}_{\text {init }}\right) ; \\
& \text { while } \mathrm{U}_{\mathrm{k}}-\mathrm{L}_{\mathrm{k}}>\varepsilon,\{ \\
& \text { pick } \mathrm{Q} \in \mathfrak{I}_{\mathrm{k}} \quad \text { such that } \phi_{\mathrm{ub}}(\mathrm{Q})=\mathrm{U}_{\mathrm{k}} ; \\
& \text { split } \mathrm{Q} \text { into } \mathrm{Q}_{\mathrm{I}} \text { and } \mathrm{Q}_{\mathrm{II}} \text { along the longest } \\
& \mathfrak{I}_{\mathrm{k}+1}:=\left(\mathfrak{I}_{\mathrm{k}}-\{\mathrm{Q}\}\right) \mathrm{U}\left\{\mathrm{Q}_{\mathrm{I}}, \mathrm{Q}_{\mathrm{II}}\right\} ; \\
& \mathrm{L}_{\mathrm{k}+1}:=\max \mathrm{Q}_{\varepsilon \mathfrak{k}+1} \quad \phi_{\mathrm{lb}}(\mathrm{Q}) ; \\
& \mathrm{U}_{\mathrm{k}+1}:=\max \mathrm{Q}_{\varepsilon \mathfrak{k}+1} \quad \phi_{\mathrm{ub}}(\mathrm{Q}) ; \\
& \mathrm{k}=\mathrm{k}+1 \\
& \}
\end{aligned}
$$$$
\text { split } \mathrm{Q} \text { into } \mathrm{Q}_{\mathrm{I}} \text { and } \mathrm{Q}_{\mathrm{II}} \text { along the longest edge ; }
$$

Where, $\mathrm{k}$ - the iteration index, $\mathfrak{I}_{\mathrm{k}}$-the list of rectangles, $\mathrm{L}_{\mathrm{k}^{-}}$the lower bound and $\mathrm{U}_{\mathrm{k}}$-the upper bound $\phi_{\text {min }}\left(Q_{\text {init }}\right)$.

Electrical Distribution Networks Planning: A significant number of studies have been devoted to optimization the distribution system using different computational methods, and the Branch and Bound algorithm of the linear programming methods has been employed to find an optimal solution to design problem.

Considering a close loop network consisting of number nodes (n) so it seems that the possible variants will be $\frac{(\mathrm{n}-1) !}{2}$. The branch and bound method is an effective math device, as it is based on implicit systematic enumeration of feasible solution set and it provides us with the optimal solution. The set of feasible solution is successively divided (branched) into smaller subsets. If minimum of cost of the distribution network is our 


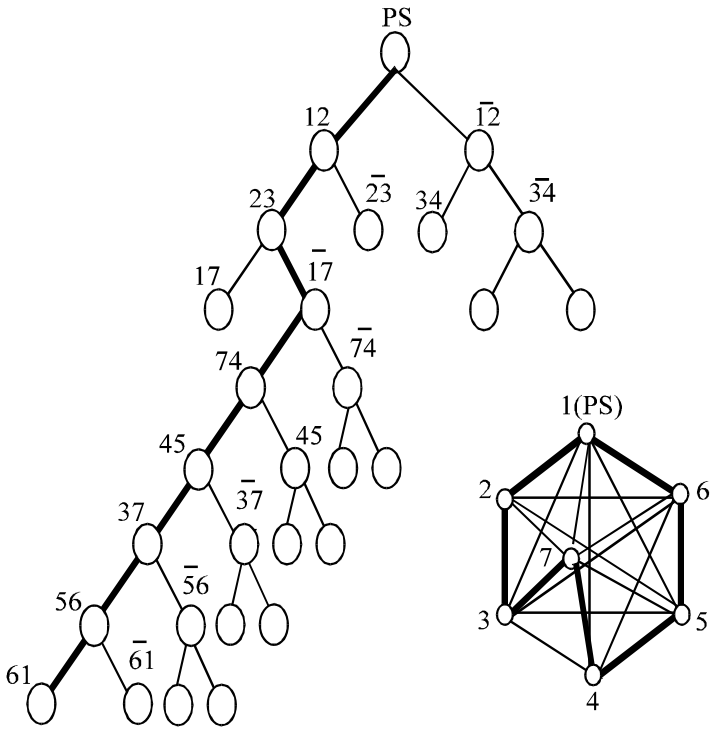

Fig. 2: The Graph and the Tree for the Branch and Bound Method

Power Source
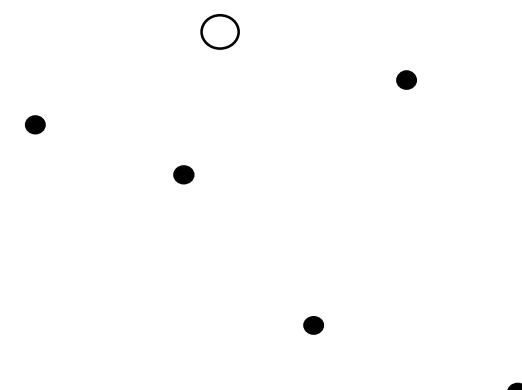

Fig. 3: Data for Planning Network

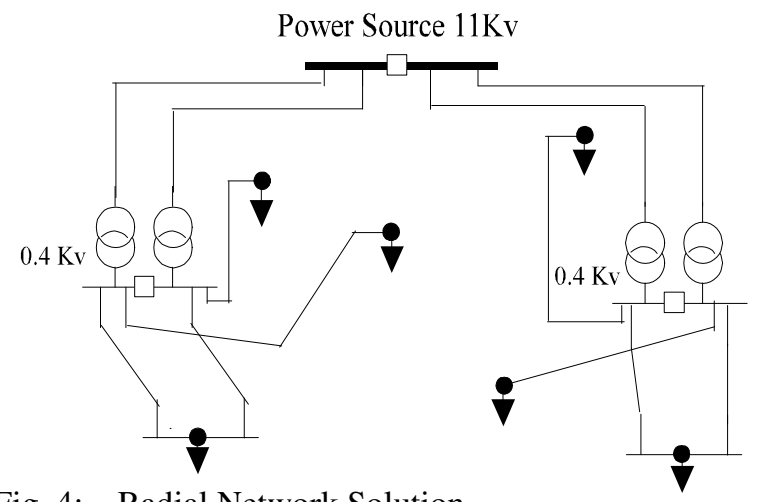

Fig. 4: Radial Network Solution

aim, then for each subset the lower bound (called simply bound) for all solution of this subset is calculated. Branching proceeds until sets with single solutions are obtained. After each division we eliminate all these subsets for which bound are greater than the cost of any solution. The best actual solution is optimal if there is no more subsets to be branched more.

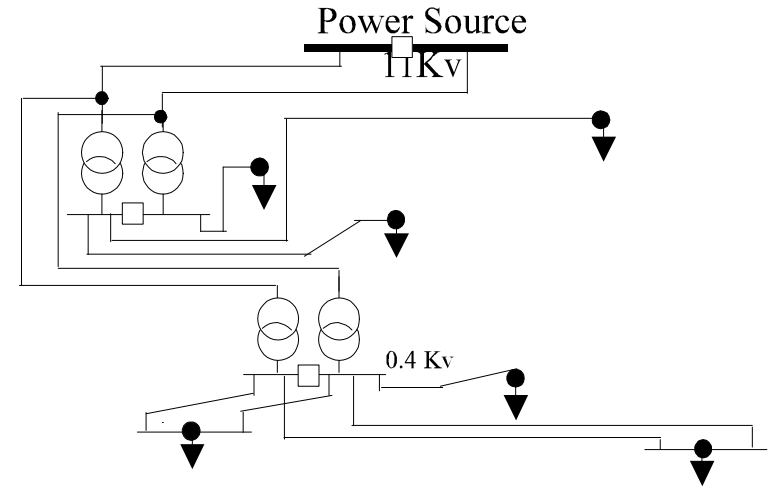

Fig. 5: Mixed Radial and Main Type Structure the Schemes

The efficiency of this methodology depends on two important factors: the branching rule or the principle of branching, and the bound calculation bases.

However, the exact efficiency improvement is very hard to pin down. It cannot be mathematically represented, as it's differs for each different problem. And the designers (design engineers) sometimes have different opinions for different reasons for the same problem and so they carry out different configurations. This makes the task more sophisticated.

For example, suppose that there are 7 distribution nodes and we wish to fit a network

The possible loop configurations are:

$$
\frac{(\mathrm{n}-1) !}{2}=\frac{6 !}{2}=360
$$

The enumeration tree and the graph can be illustrated in Fig. 2 (note that PS - the power source).

Suppose that the nodes are placed as in Fig. 3 and we have to optimize the configuration between these nodes to fulfill with the up formulated objectives in Fig. 1. Applying the Bound and Branch method, the solution was a pure radial networks solution the schemes in Fig. 4. Changing the branching rule and the bound calculation bases, we get solution of network with a mixed radial and main type structure the schemes in Fig. 5. Both of the schemes are optimal for the given nodes.

\section{CONCLUSION}

* The branch and bound methodologies are an effective tool for solving such problems, as the set of feasible solution is successively divided into subsets. The branching is continued till the desired solution sets are obtained.

* These methods are adaptive mathematical tools as they can easily carry any change or expansions in the problem data or in its conditions. 
* The optimal configuration by using the branch and bound methodologies strongly depends on the branching rule and on the bound calculation bases.

\section{REFERENCES}

1. Pansini Anthony, J., 1992. Electrical Distribution Engineering. The Fairmont Press, $2^{\text {nd }}$ Edn.

2. Viktoria Neimane, 2001. On development planning of electricity distribution network. Ph. D Thesis. Royal Institute of Technology, Stockholm.

3. Kuwabara, H. and K. Nara, 1996. Multi-year and multi-state distribution system expansion planning by multi-stage branch exchange. IEEE/PES Summer Meeting,

4. Jalal Abdallah and Abdallah Al-Thamir, 2004. The economic-environmental neural network model for electrical power dispatching. J. Applied Sci., 4: 340-343.
5. Gupta, B.R., 1996. Power System Analysis and Design. Wheeler Publishing.

6. Hindi, K.S., 1997. Design of Low-voltage Distribution networks, a mathematical programming method. Proc. IEE, 124: 54-58.

7. Boyd, S., A. Ghosh and A. Magnani, 2003. Branch and bound methods. Notes for the EE392o. Stanford University.

8 Vaziri, M., K. Tomsovic and G. Turan, 2002. Distribution expansion problem revisited. Part 1 Report- Department of EECS. Washington State University, USA.

9. Belgain Turkay, 1998. Distribution system planning using mixed integer programming. Elektrik, 6: 1. Istanbul, Turkey. 